\title{
A Fast and Accurate Iris Localization Technique for Healthcare Security System
}

\author{
Alaa S. Al-Waisy, Rami Qahwaji, Stanley Ipson, Shumoos Al-Fahdawi \\ School of Computing, Informatics \& Media, University of Bradford. \\ Bradford City, UK. \\ King alaa87@yahoo.com, R.S.R.Qahwaji@bradford.ac.uk, S.S.Ipson@Bradford.ac.uk, \\ S.T.Hammadi@student.bradford.ac.uk.
}

\begin{abstract}
In the health care systems, a high security level is required to protect extremely sensitive patient records. The goal is to provide a secure access to the right records at the right time with high patient privacy. As the most accurate biometric system, the iris recognition can play a significant role in healthcare applications for accurate patient identification. In this paper, the corner stone towards building a fast and robust iris recognition system for healthcare applications is addressed, which is known as iris localization. Iris localization is an essential step for efficient iris recognition systems. The presence of extraneous features such as eyelashes, eyelids, pupil and reflection spots make the correct iris localization challenging. In this paper, an efficient and automatic method is presented for the inner and outer iris boundary localization. The inner pupil boundary is detected after eliminating specular reflections using a combination of thresholding and morphological operations. Then, the outer iris boundary is detected using the modified Circular Hough transform. An efficient preprocessing procedure is proposed to enhance the iris boundary by applying 2D Gaussian filter and Histogram equalization processes. In addition, the pupil's parameters (e.g. radius and center coordinates) are employed to reduce the search time of the Hough transform by discarding the unnecessary edge points within the iris region. Finally, a robust and fast eyelids detection algorithm is developed which employs an anisotropic diffusion filter with Radon transform to fit the upper and lower eyelids boundaries. The performance of the proposed method is tested on two databases: CASIA Version 1.0 and SDUMLA-HMT iris database. The Experimental results demonstrate the efficiency of the proposed method. Moreover, a comparative study with other established methods is also carried out.
\end{abstract}

Keywords-Iris Localization, Iris Segmentation, Radon Transform, Circular Hough Transform, SDUMLA-HMT database, CASIA database.

\section{INTRODUCTION}

One of the most sensitive types of data is the medical records that have a quite serious effect on the patient's life. Increasing the electronic access to these sensitive records can raise many issues such as, the misuse and access control to this data. Therefore, security is a key concern in healthcare applications to protect the patient's data that have direct threat to the patient's life. Biometric systems can play a significant role in healthcare applications by facilitating secure access to the medical records and accurate patient identification [1]. A biometric system is a classification and recognition system that works on measuring and analysing the human's physiological and behavioural characteristics for identifying and/or authenticating a user's identity uniquely. In a biometric system, these characteristics constitute a substantial and a strong link between the users and their identity [2]. In this regard, the biometric system based on the iris trait is considered as the most accurate and highly reliable system for personal authentication and/or identification [3]. The iris trait has a number of features that make it convenient to be applied in a high confidence biometric system. Firstly, the iris trait represents a highly protected part of the human eye; it is located between the pupil and the sclera. This position makes the iris trait completely isolated and protected from the external environmental conditions [4],[5]. Secondly, due to their high degree of the randomness, iris patterns are regarded as unique. No two humans have been observed with the same iris patterns, even identical twins or the right and left eye of the same subject. Finally, unlike other biometric traits such as fingerprints, which can be easily forged, it is very difficult to tamper with the iris patterns, even with a surgery, which is always coupled with high risks. This makes the iris trait secure against fraudulent methods and spoofing attacks by an imposter [6]. Typically, the iris recognition system consists of four main stages: image acquisition, preprocessing, feature extraction and classification. The image preprocessing stage can be divided further into three sub-steps: iris localization, iris normalization and iris enhancement. Iris localization refers also to the iris segmentation, which is a crucial step to build an efficient, robust and real-time iris recognition system. The main aim of the iris localization is to detect the iris region from the background and all surrounding features such as sclera, pupil, eyelashes, eyelids, eyebrows and specular reflections. Accurate iris localization can be achieved by delimiting the inner border between the pupil and iris and the outer border between the iris and sclera. However, the task becomes more challenging, due to the portions of the iris region occluded by eyelids and eyelashes. In addition, the small difference of the intensity levels between the iris and the sclera regions makes detecting the outer boundary more difficult compared to the detection of the inner boundary. Finally, varying illumination conditions that can affect the appearance of the iris patterns could greatly influence the iris segmentation outcome [7],[8],[9]. In this paper, an efficient and automatic method is proposed for the inner and outer iris boundary localization, which addresses the main concerns in many previous methods for instance, the computational cost and high accuracy. Firstly, the pupil boundary is detected after eliminating the specular 
reflections using a simple thresholding technique with the morphological operations. Then, the outer iris boundary is detected using the generated edge map along with the modified Circular Hough transform. At this stage, an efficient preprocessing procedure is proposed to enhance the iris boundary by applying 2D Gaussian filter and Histogram equalization processes. Finally, a robust and fast eyelids detection algorithm is developed by employing an anisotropic diffusion filter with Radon transform to fit the upper and lower eyelids boundaries. The performance of the proposed method has been tested on two databases: CASIA Version 1.0 and SDUMLA-HMT iris database. This paper is organized as follows: some related works are discussed in section 2 . Section 3 provides an overview of the proposed approaches. The proposed iris localization method is presented in Section 4. Section 5 presents the experimental results of the proposed method. Finally, conclusions and future research directions are drawn in the last section.

\section{RELATED WORKS}

Recently, iris segmentation has been receiving increased attention, due to its direct effects on the performance and accuracy of the iris recognition system. In this regard a number of iris segmentation algorithms have been proposed in the past. The first implemented iris segmentation was in 1994, when John Daugman [10] proposed an integro-differential operator for detecting iris boundaries and eliminating possible extraneous features such as the eyelids and eyelashes. This operator calculates the partial derivative of the average intensity of circle points taking into account the increasing radius (r), followed by convolving the operator with a Gaussian filter. Then, the center and radius of the iris boundaries are identified by computing the maximum difference between outer and inner circle. Finally, the eyelids are detected by using the parabolic curve fitting approach. The main limitation of Daugman's method is that the Integrodifferential operator can be easily affected by local gradient maximum where light spots can change the gradient greatly, which results in inaccurate iris localization. Wildes [11] proposed a Circular Hough transform based filtering and voting procedure to localize the two circular boundaries of the iris. An edge detection filter applied to the eye image generates an edge image map which is subjected to the Hough transform voting procedure to find the desired edge map contour. The center coordinates and radius of the circle with maximum number of votes is detected as an iris boundary. The main limitation of this method is its computational complexity which makes it inappropriate to meet the real-time requirements. Xiaofu H. and Pengfei S. [12] proposed an iris localization algorithm for hand-held capture devices. The inner boundary is detected using a geometrical method where the bottom point of pupil circle is used as a reference point for pupil detection because it is not affected by the top eyelashes and eyelid. Then, the outer boundary is detected using the Hough transform. Arun R. and Samir S. [13] proposed a new iris segmentation algorithm using Geodesic Active Contours to detect the iris region from input eye image. Firstly, the pupil is detected by applying a median filter on the eye image.
This is followed by a simple thresholding method and circle fitting procedure. Then, an iterative process based on the GAC is employed to detect the outer boundary as well as the eyelid contour. The performance of the segmentation algorithm was tested by applying the multiple Gabor filters on the normalized iris template to observe its performance in the recognition task. The results obtained were $94 \%$ success on the CASIA version 1 database. Cui et al [14] proposed a segmentation algorithm based on scaling the eye image using the Haar wavelet, followed by applying a Hough transform and integro-differential operator to detect the inner and outer boundaries, respectively. Histogram and parabolic arc based approaches are used to detect lower and upper eyelids, respectively.

\section{OVERVIEW OF THE PROPOSED APPROACHES}

Since the proposed iris segmentation algorithm is based on the modified Circular Hough transform and Radon transform, they are briefly reviewed here.

\section{A. Modified Circular Hough Transform}

The standard Circular Hough transform (CHT) works on detecting the circular shapes of a given radius within the image. The edge map of the eye image is generated by computing the first derivatives of intensity values. Each point in the edge map donates a circle of radius $\boldsymbol{r}$ and center $\left(\boldsymbol{x}_{c}, \boldsymbol{y}_{c}\right)$ to an output accumulator array. Then, a voting procedure is used to find the largest peak in the resulting accumulator array in the parameter space, which corresponds to the circle best defined by the edge points [15]. Considering the obtained set of edge points as $\left(\boldsymbol{x}_{\boldsymbol{i}}, \boldsymbol{y}_{\boldsymbol{i}}\right), \boldsymbol{i}=\mathbf{1 , 2}, \ldots, \boldsymbol{n}$. The CHT for fitting a circle with radius $\boldsymbol{r}$ and center coordinates $\left(\boldsymbol{x}_{c}, \boldsymbol{y}_{c}\right)$ is defined as follows:

$$
H\left(x_{c}, y_{c}, r\right) \sum_{i=1}^{n} h\left(x_{i}, y_{i}, x_{c}, y_{c}, r\right)
$$

Where

$h\left(x_{i}, y_{i}, x_{c}, y_{c}, r\right)=\left\{\begin{array}{lr}1, & \text { if } g\left(x_{i}, y_{i}, x_{c}, y_{c}, r\right)=0 \\ 0, & \text { otherwise }\end{array}\right.$

And the parametric function $\mathrm{g}$ is defined as follows:

$$
g\left(x_{i}, y_{i}, x_{c}, y_{c}, r\right)=\left(x_{i}-x_{c}\right)^{2}+\left(y_{i}-y_{c}\right)^{2}-r^{2}
$$

The value of the function $g$ of each point is equal to $\mathbf{1}$, if it is located over the circle with parameters $\left(\boldsymbol{x}_{\boldsymbol{c}}, \boldsymbol{y}_{\boldsymbol{c}}, \boldsymbol{r}\right)$ by (2). Finally, the voting procedure is implemented in the Hough space to detect the correct circle. A number of modifications have been applied to the CHT to either improve the localization rate or to decrease its computational complexity. In this paper, the modified CHT by Atherton and Kerbyson [16] is employed to improve the inner and outer boundary localization rate and to reduce the execution time, using a complex phase coding to produce a complex accumulator 
array.The word complex here refers to the real and imaginary numbers in the generated accumulator array. In the modified CHT, phase is used to code for the radius of the circle, where the phase is relative to the distance travelled far from the edge points. After projecting the edge points along a line in the direction of the edge orientation, their complex values are added to the accumulator array. The projections of the edge points lying on the same circle will be intersected at a common point and within the same phase which means they have the same distance from this intersection point. While, edge projections are not lying on a circle border (e.g. noise edge points) will be in a random phase and tend to be discarded. In other words, the contribution of each edge point to the accumulator array is within the phase if this point is lying on the circle and out of the phase elsewhere. Finally, the circle parameters are obtained where the center coordinates are represented by the positions of the peaks in the magnitude of the accumulator array, while the radius is represented by the phase at the peak position. More details can be found in [16].

\section{B. Radon Transform}

In 1917, an integral transform was introduced by the Austrian mathematician named Johann Radon. The Radon transform, as it became known, is now an important mathematical tool that is widely used in many medical imaging, geophysical imaging and radar imaging applications [17]. The Radon transform is a set of 1D projections of a given $2 \mathrm{D}$ function $\boldsymbol{f}(\boldsymbol{x}, \boldsymbol{y})$. In this case, the given 2D function is an image, and the projection is a set of line integrals. These line integrals are computed from multiple resources by the Radon transform in a certain angle. Generally, the Radon transform of the 2D function $\boldsymbol{f}(\boldsymbol{x}, \boldsymbol{y})$ is the line integral of $\boldsymbol{f}$ at an angle $\theta$, that parallel to the $\boldsymbol{y}^{\prime}$-axis defined as follows:

$$
\begin{gathered}
R_{\theta}\left(x^{\prime}\right)=\int_{-\infty}^{\infty} f\left(x^{\prime} \cos \theta-y^{\prime} \sin \theta, x^{\prime} \sin \theta\right. \\
\left.+y^{\prime} \cos \theta\right) d y^{\prime}
\end{gathered}
$$

Where $\boldsymbol{x}^{\prime}$ and $\boldsymbol{y}^{\prime}$ are computed as follows:

$$
\left[\begin{array}{l}
x^{\prime} \\
y^{\prime}
\end{array}\right]=\left[\begin{array}{rr}
\cos \theta & \sin \theta \\
-\sin \theta & \cos \theta
\end{array}\right]\left[\begin{array}{l}
x \\
y
\end{array}\right]
$$

The Radon transform and the Hough transform are similar in the sense they map the data points from the image space to a parameter space. However, they differ in their mapping form. The Radon transform derives a point in parameter space from image space and this mapping is known as the reading paradigm. The Hough transform, on the other hand, depends on the writing paradigm in mapping the data points from image space to the parameter space. Moreover, unlike the Hough transforms, the radon transform has a well formed mathematical construct [18]. In this paper, the Radon transform is employed to detect the parts of the upper and lower eyelids' boundaries that can be modelled as a line without losing important information from the iris region.

\section{THE PROPOSED SYSTEM}

As mentioned previously accurate iris localization plays a significant role in improving the performance of iris recognition systems. The goal of the iris localization is the precise detection of both inner and outer boundaries of the iris region. However, a number of issues need to be addressed before and after the detection of these two boundaries, such as detecting and eliminating the reflection spots, eyelashes and eyelids. In this paper, the proposed iris localization method can be divided broadly into four stages: specular reflection removal, pupil localization, iris localization and eyelid and eyelash detection.

\section{A. Specular Reflection Removal}

One of the major issues in the iris segmentation and recognition system is the presence of reflection spots, which can result in inaccurate iris localization and thereby lead to poor iris recognition system performance. In this case, these spots need to be detected and isolated to get a clean iris pattern that can contribute to increase the recognition rate. In this work, a reflection mask $\left(\boldsymbol{R}_{\boldsymbol{m}}\right)$ is calculated to detect and remove the reflection spots along the eye image in two steps. Firstly, an adaptive threshold is applied, and if the intensity of the pixel $I(x, y)$ is greater that the determined threshold $(\boldsymbol{T})$, then it will be considered as a reflection noise.

$$
R_{m}(x, y)= \begin{cases}1, & I(x, y)<T \\ 0, & \text { otherwise }\end{cases}
$$

Secondly, a morphological dilation operation is applied using square shaped structure element whose width is 5 pixels for perfect detection and elimination to the reflection spots. Finally, the specular reflection spots in the eye image are painted using a defined reflection mask and a roifill Matlab function. As shown in Fig.1, the specular reflections spots in the eye image have been removed efficiently; thereby this could contribute in improving the quality of iris patterns and the performance of the iris localization method.

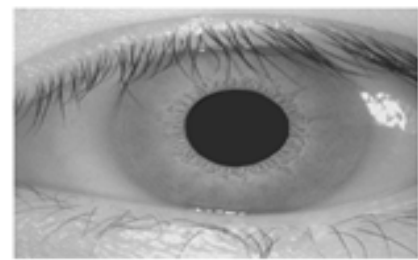

(a)

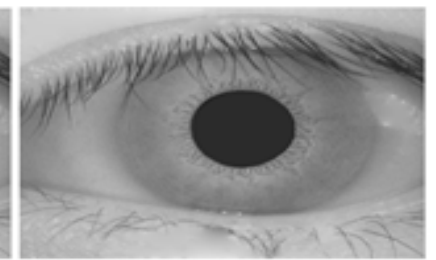

(b)
Fig.1. Specular reflection removal stage: (a) the original eye image and (b) the output image.

\section{B. Pupil Localization}

In this paper, the inner boundary (pupil-iris boundary) is detected before the outer boundary (iris-sclera boundary), due to the fact that the pupil region is the darkest region in the eye image and can be detected easily. Moreover, this can contribute to improving the accuracy and the speed of detecting the outer boundary as will be explained later on. The 
pupil localization is carried out by transforming the grayscale eye image into a binary image using the Hysteresis thresholding method. In this method, all pixels that have values above upper threshold $\boldsymbol{T}_{u p}$ are marked as edge points. In addition, all the adjacent pixels to these edge points with values greater than lower threshold $\boldsymbol{T}_{\text {low }}$ are marked as edge points as well. In this method, eight connectivity is used to detect connected regions to each edge point. As shown in Fig.2 (b), there could exist some noise present in the binary image, due to other dark regions, such as eyelashes and eyelids. A morphological erosion operation using a disk shaped structure element of one pixel radius is applied to eliminate such noise. This is followed by discarding all the connected components smaller than 80 pixels in order to produce the final binary image. As shown in Fig.2 (c), the pupil region is almost completely detected in the binary image. Since it can be modelled as a circle, the pupil is detected correctly by employing a modified Circular Hough transform to obtain the center coordinates and radius of the pupil circle. Fig.2 (d) shows the detected inner boundary of the iris region.

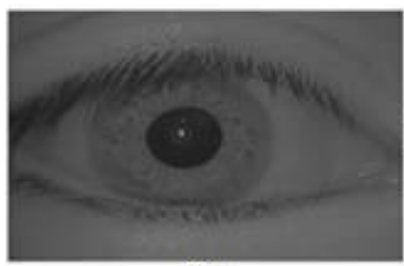

(a)



(c)

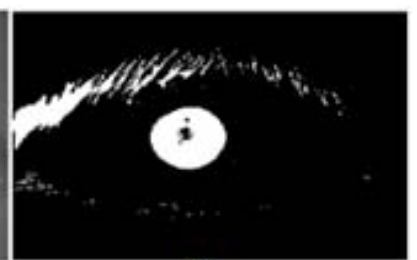

(b)

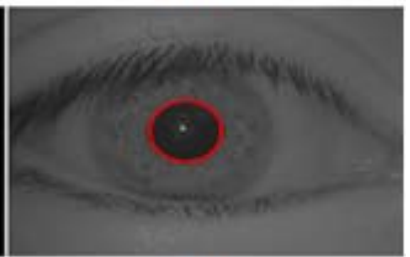

(d)
Fig. 2. Pupil localization stage: (a) The input image, (b) applying the Hysteresis thresholding method, (c) the output of the morphological operation (d) the localized pupil boundary.

\section{Iris Localization}

The most challenging stage of the iris region localization model is detecting the outer boundary between the iris and sclera, for several reasons. Firstly, no clearly defined border exists between the iris and sclera because of the low variation in intensity between them. Secondly, the upper part of the iris is occluded by upper eyelid and eyelashes in most cases. In this paper, the edge map is generated using a Canny edge detector followed by the modified CHT to localize the iris outer boundary. Each edge point in the edge map casts a vote in the Hough transform space and produces a circle of radius $r$ to the output accumulator array. The circle with the highest number of votes is then chosen. However, the main concern here is that the Hough transform is computationally expensive and increasing the number of unnecessary edge points can result in inaccurate iris localization. Therefore, to reduce the search space the eye image needs to be enhanced before and after generating the edge map. In this work, a 2D Gaussian filter is employed in order to smooth the eye image and reduce noise, as shown in Fig.3 (b). The Gaussian filter is a low-pass filter, whose $2 \mathrm{D}$ filter coefficients are computed as follows:

$$
G(x, y)=\frac{1}{2 \pi \sigma^{2}} e^{-\frac{x^{2}+y^{2}}{2 \sigma^{2}}}
$$

Where $\boldsymbol{x}$ and $\boldsymbol{y}$ are the distances from the origin along the horizontal and vertical axes, and $\boldsymbol{\sigma}=\boldsymbol{0 . 8}$ is the standard deviation of the Gaussian distribution. After that, the Histogram equalization is used to enhance the contrast between the iris and sclera region, and to make detecting the iris outer boundary easier, as shown in Fig.3 (c). In the next step, an edge map of the eye image is generated by applying the Canny edge detector. As can be seen in Fig.3 (d), there are a lot of unnecessary edge points that need to be identified and eliminated to improve the performance of the CHT. In this paper, based on prior knowledge that the center of the iris and pupil are relatively close, and to the established fact in [19],[20] that the ratio of iris radius/pupil radius fall between 4 and 1.75 , a circle using the pupil center is drawn and the pixels within the circle are set to zero. The radius of the drawn circle was selected empirically to be $\left(\boldsymbol{r}_{\text {pupil }}+\boldsymbol{6}\right)$ for all the datasets. This procedure eliminates edge points within the iris region that represent the pupil boundary and iris tissue without the risk of affecting the iris outer boundary. Then, all the connected components smaller than 99 pixels are excluded, as shown in Fig.3 (e). These parameters are adjusted only once for all the databases considered. Finally, the center coordinates and radius of the iris circle are obtained by applying the modified CHT, as shown in Fig.3(f).

\section{Eyelids and Eyelashes Detection}

This stage of detecting the eyelids boundaries and the eyelashes has a significant role in either increasing or degrading the performance of the iris localization model and the iris recognition system. Therefore, these artefacts need to be identified and eliminated to obtain a clean iris template. However, detecting the eyelids boundaries is a challenging problem as they can be occluded by the eyelashes in many cases. A number of eyelids detection algorithms have been proposed in the literature as described in [21] where the boundary between the eyelids and iris is modeled as a parabolic arc or line. In this work, an efficient and robust algorithm is proposed by fitting these boundaries as straight lines where the line form has fewer parameters than the parabolic arc and thereby requires less processing time. Firstly, an anisotropic diffusion filter [22] is applied on the eye image in order to enhance the eyelids boundaries and reduce the eyelashes effect, as shown in Fig.4. The anisotropic diffusion filter can be defined as follows:

$$
\begin{gathered}
I_{i, j}^{t+1}=I_{i, j}^{t}+\lambda\left[c_{N} \cdot \nabla_{N} I+c_{S} \cdot \nabla_{S} I+c_{E} \cdot \nabla_{E} I+\right. \\
\left.c_{W} \cdot \nabla_{W} I\right]_{i, j}^{t}
\end{gathered}
$$




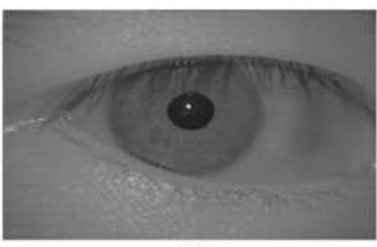

(a)

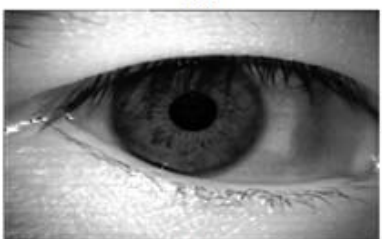

(c)

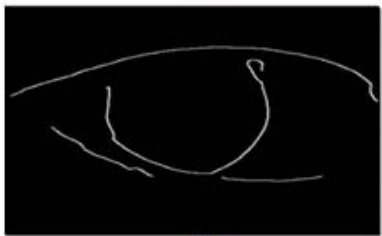

(e)

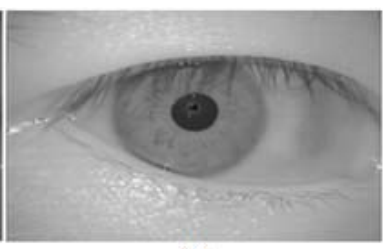

(b)



(d)

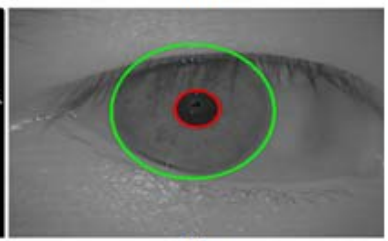

(f)
Fig. 3. Iris localization stage: (a) The input image, (b) Applying the 2D Gaussian filter, (c) Histogram equalization is employed, (d) Edge map, (e) Further processing for removing noise and (f) The final detected iris boundaries.

Where $I$ is the eye image, $0 \leq \lambda \leq 1 / 4, c$ refers to the conduction coefficients that updated each iteration as a function of the brightness gradient, $\boldsymbol{t}$ refers to the iteration index, and $\boldsymbol{\nabla}$ points to the nearest neighbor differences in all the directions $\boldsymbol{N}, \boldsymbol{S}, \boldsymbol{E}$ and $\boldsymbol{W}$, as follows:

$$
\begin{aligned}
& \nabla_{N} I_{i, j}=I_{i-1, j}-I_{i, j} \\
& \nabla_{S} I_{i, j}=I_{i+1, j}-I_{i, j} \\
& \nabla_{E} I_{i, j}=I_{i, j+1}-I_{i, j} \\
& \nabla_{W} I_{i, j}=I_{i, j-1}-I_{i, j}
\end{aligned}
$$

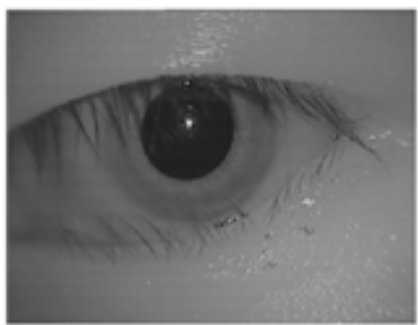

(a)

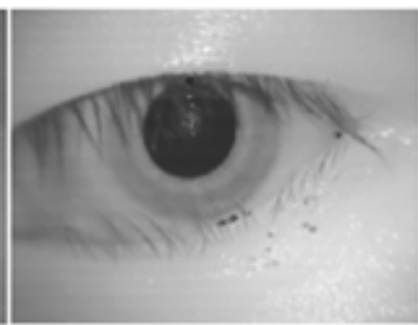

(b)
Fig. 4. (a) An occluded eye image and (b) the output of the anisotropic diffusion filter.

In this work, $\boldsymbol{\lambda}=\mathbf{0 . 2 1}$ and $\boldsymbol{t}=\boldsymbol{6}$. Secondly, the search time for the Radon transform is reduced by extracting the iris and dividing it into upper and lower parts, as shown in Fig.5 (a) and (b). This is followed by generating the edge map of each part using a modified Canny edge detector where only the horizontal edges are considered, as shown in Fig.5 (c). Finally, the Radon transform is implemented to fit the upper and lower eyelids boundaries. In the proposed method, there is no line to fit, if the maximum of the Radon transform space is less than the predefined threshold, which means that the iris region is not occluded by the eyelids. Generally, the eyelashes can be divided into types: separable and multiple eyelashes that grow along the eyelid boundaries. In addition, it is observed that the eyelashes have a lower intensity values than the iris region. In this paper, a simple thresholding technique is employed to detect eyelashes carefully to avoid remove important iris information, as shown in Fig.5 (d).

\section{EXPERIMENTAL RESULTS}

In this section, the performance of the proposed iris localization algorithm was evaluated using two databases, the CASIA Version 1.0 database [23] and the SDUMLA-HMT iris database [24]. The CASIA database consists of 756 images captured from 108 persons with 7 images for each one. The images in this database were collected in two sessions with some irises occluded by the upper and/or lower eyelids. All images are stored in "BMP" format with an image size of $(320 \times 280)$ pixels. The SDUMLA-HMT iris database is composed of 106 persons with each one providing 10 images (e.g. 5 images for each eye). Therefore, the total is 1,060 images taken using an intelligent iris capture device with a distance between the device and the eye between $6 \mathrm{~cm}$ and $32 \mathrm{~cm}$. The images are stored in grayscale level and "BMP" format with an image size of $(768 \times 576)$ pixels. To the best of our knowledge, this is the first work that uses the SDUMLAHMT iris database for evaluating an iris segmentation method. In this work, the iris localization is considered as accurate only if two conditions are satisfied. Firstly, the inner and outer boundaries are correctly localized. Secondly, the upper and the lower eyelids are correctly detected, as shown in Fig.6. If one of the above conditions is not provided, then the iris localization is not precise, as illustrated in Fig.7. The accuracy rate of the proposed iris localization method was computed by dividing the number of correctly localized iris images with the number of all the iris images in the database. In addition, the localization time was computed by implementing the proposed algorithm on a PC laptop with Windows 8.1 operating system, $6 \mathrm{~GB}$ of RAM and a $1.80 \mathrm{GHz}$ Core i5-3337U CPU. The proposed algorithm was coded using MATLAB R2013b. Table I. shows the localization rate and the execution time per image for different databases. As shown in Table I, a high accuracy rate and fast iris localization are obtained by applying the proposed method on the CASIA and SDUMLA-HMT database. The proposed method managed to correctly localize the iris boundaries from 749 out of 756 eye images in the CASIA database, while 517 and 511 eye images are correctly segmented out of 530 in the SDUMLA-HMT database for the right and left eye, respectively. Moreover, using the Radon transform in the eyelids boundary detection increases the speed of the proposed method, which has the advantage over other fitting algorithms such as parabolic 


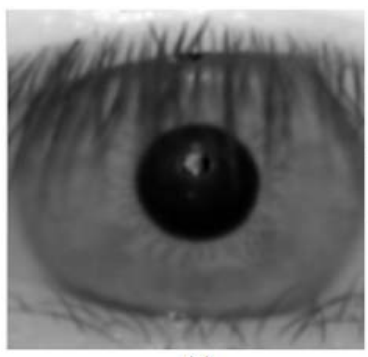

(a)
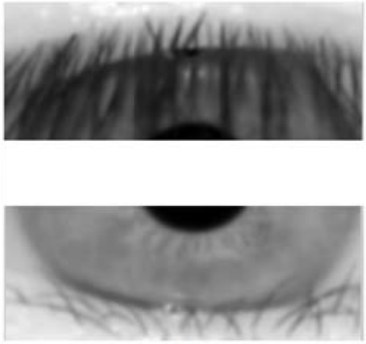

(b)

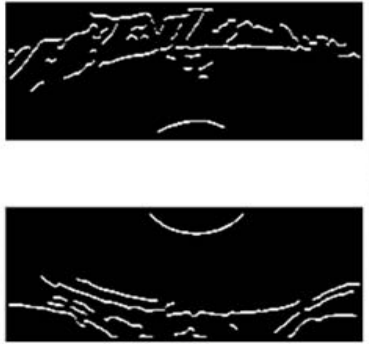

(c)

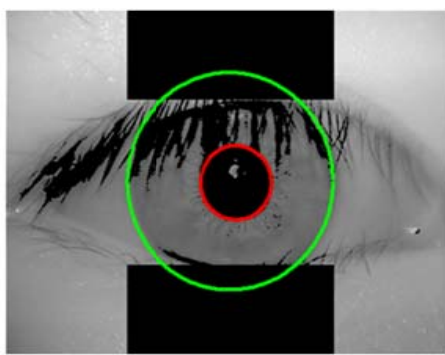

(d)

Fig.5. Eyelids and Eyelashes Detection: (a) The iris boundary region, (b) The top and bottom parts of iris region, (c) The edge map the top and bottom parts, (d) The final detected eyelids and eyelashes.

Hough transform. In this work, the main causes of the error iris localization are due to the weak contrast between the iris and sclera regions, the presence of eyelashes that cover the iris and/or the eyelids and the low illumination. However, in most of the failing situations the proposed method is still able to correctly detect at least one of the iris boundaries. For further evaluation, the performance of the proposed method is compared with other established algorithms in Table II. The performance of the proposed method is seen to outperform all these alternative methods in terms of accuracy and in terms of speed in two out of three cases where this information is available.

TABLE I. THE ACCURACY AND EXECUTION TIME OF THE PROPOSED IRIS LOCALIZATION METHOD.

\begin{tabular}{|c|c|c|}
\hline The Database Name & $\begin{array}{c}\text { Accuracy } \\
\text { Rate } \%\end{array}$ & $\begin{array}{c}\text { Average } \\
\text { Runtime (s) }\end{array}$ \\
\hline CASIA Version 1.0 & 99.07 & 0.9 \\
\hline $\begin{array}{c}\text { SDUMLA-HMT (Right } \\
\text { Eye) }\end{array}$ & 97.55 & 1.7 \\
\hline $\begin{array}{c}\text { SDUMLA-HMT (Left } \\
\text { Eye) }\end{array}$ & 96.42 & 1.7 \\
\hline
\end{tabular}

TABLE II. COMPARISON OF THE PROPOSED METHODS WITH PREVIOUS APPROACHES BASED ON CASIA DATABASE.

\begin{tabular}{|l|c|c|}
\hline \multicolumn{1}{|c|}{ The Methodology } & $\begin{array}{c}\text { Accuracy } \\
\text { Rate } \%\end{array}$ & $\begin{array}{c}\text { Average } \\
\text { Runtime (s) }\end{array}$ \\
\hline Daugman [10] & 98.6 & 6.56 \\
\hline Libor Masek [25] & $\mathbf{8 3}$ & 9.37 \\
\hline Liam and et al [26] & $\mathbf{6 4 . 6 4}$ & NA \\
\hline Mahmoud and Ali [27] & 97.50 & NA \\
\hline Hentati and et al [28] & 92.6 & $\mathbf{0 . 2 9 0}$ \\
\hline GuangZhu and et al [29] & $\mathbf{9 8 . 4 2}$ & NA \\
\hline The proposed method & $\mathbf{9 9 . 0 7}$ & $\mathbf{0 . 9}$ \\
\hline
\end{tabular}

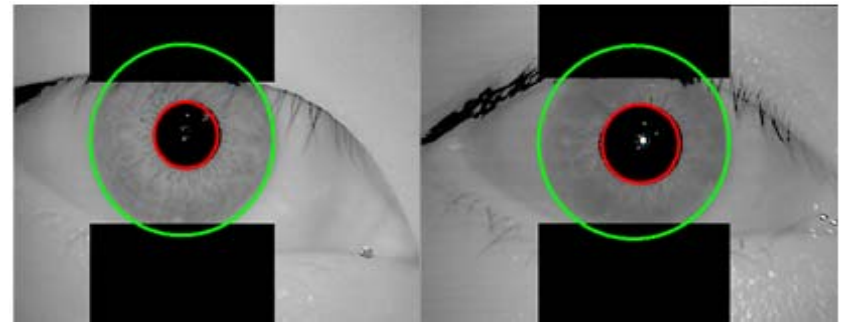

Fig.6. Examples of correct localized iris region.

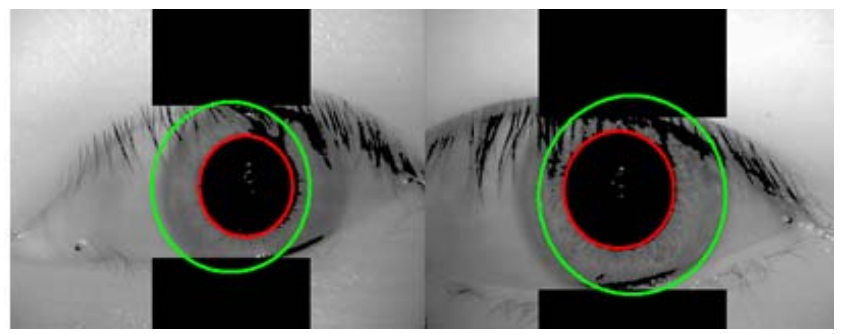

Fig.7. Examples of failed iris localization.

\section{CONCLUSIONS AND FUTURE WORK}

In this work, the cornerstone towards building a fast and robust iris recognition system for healthcare applications is addressed by proposing an efficient and fast iris localization method to correctly detect the iris region from the extraneous features such as eyelashes, eyelids, pupil and specular reflections. In general, the overall accuracy of the proposed method has been increased due to the proposed modifications made on the generated edge map, which results in less processing time for detection the pupil and iris boundaries. Moreover, the detection rate of the upper and lower eyelids boundaries has been improved without losing an important information from the iris region. Finally, the effectiveness and robustness of the proposed method has been demonstrated by testing it on two datasets and compare the results obtained with other previous methods. For the future work, the performance of the proposed method will be observed using large datasets with more noisy images. In addition to include the proposed method in an iris recognition system using one of the deep learning approaches (e.g. Convolutional Neural Network, Autoencoders and etc.) in order to evaluate its influence on the precision of the iris recognition system in the healthcare applications. 


\section{REFERENCES}

[1] S. J. Al-Hijaili1and M. AbdulAziz, "Biometrics in Health Care Security System, Iris-Face Fusion System ," Int. J. Acad. Res., vol. 3, no. 1, pp. 11-19, 2011.

[2] K. Nandakuma, "Multibiometric Systems: Fusion Strategies and Template Security," PhD thesis, Department of Computer Science and Engineering, Michigan State University, Michigan State University, 2008.

[3] R. Islam, "Feature and Score Fusion Based Multiple Classifier Selection for Iris Recognition," Hindawi Publishing Corporation, Computational Intelligence and Neuroscience, 2014.

[4] W. Aydi, N. MASMOUDI and L. KAMOUN, "Improved Masek approach for iris localization," Microelectronics (ICM), International Conference on,IEEE, 2011, pp. 1-5

[5] R. Qahwaji and R. Green," Detection of Closed Regions in Digital Images.", The International Journal of Computers and Their Applications, vol.8, no.4, pp. 202-207,2001

[6] A. Das and R. Parekh, "Iris Recognition using a Scalar based Template in Eigen-space," International Journal of Computer Science and Telecommunications, vol. 3, no. 5, pp. 74-79, May 2012.

[7] Q. Tian, H. Qu, Z. Lanfang, and R. Zong, "Personal Identity Recognition Approach Based on Iris Pattern," State art Biometrics, pp. 1-326, 2011.

[8] M.Boyd, D. Carmaciu, F. Giannaros, T, Payne, and W. Snell, "Iris Recognition," MSc Computing Science Group Project, Imperial College London, March 2010.

[9] R. Qahwaji and T. COLAK, "Neural Network-based Prediction of Solar Activities.", CITSA2006: Orlando. pp. 4-7, 2006.

[10] J. G. Daugman, "High confidence visual recognition of persons by a test of statistical independence," IEEE Trans. Pattern Anal. Mach. Intell., vol. 15, no. 11, pp. 1148-1161, 1993.

[11] R. P. Wildes, J. C. Asmuth, G. L. Green, S. C. Hsu, R. J. Kolczynski, J. R. Matey, and S. E. McBride, "A system for automated iris recognition," Proc. IEEE Work. Appl. Comput. Vis., pp. 121-128, 1994.

[12] X. He and P. Shi, "A new segmentation approach for iris recognition based on hand-held capture device," Pattern Recognit., vol. 40, no. 4, pp. 1326-1333, 2007

[13] A. Ross and S. Shah, "Segmenting non-ideal irises using geodesic active contours," Biometric Consortium Conference, 2006 Biometrics Symposium: Special Session on Research at IEEE, September, 2006.

[14] J. Cui, Y. Wang, T. Tan, L. Ma and Z. Sun "A fast and robust iris localization method based on texture segmentation," Defense and Security. International Society for Optics and Photonics,, pp. 401-408, 2004

[15] R. P. Wildes, "Iris recognition: an emerging biometric technology," Proc. IEEE, vol. 85, no. 9, pp. 1348-1363, 1997.

[16] T. Atherton and D. Kerbyson, "The coherent circle Hough transform," In BMVC, pp. 1-10. 1993.

[17] M. Alexandra,"On Radon Transform of Sampled Function," PhD Thesis, Department of Engineering Sciences, Harvard Univrsirty, Harvard, June 2003.

[18] M. V. Ginkel, C. L. Luengo Hendriks, and L. J. Van Vliet, "A short introduction to the Radon and Hough transforms and how they relate to each other," Quantitative Imaging Group, Imaging Science \& Technology Department, TU Delft, pp. 1-11, 2004.

[19] T. a. Camus and R. Wildes, "Reliable and fast eye finding in close-up images," In Pattern Recognition, Proc. 16th Int. Conf. on, vol. 1, 2002.

[20] A. Zaim, M. Quweider, J. Scargle, J. Iglesias, and R. Tang, "A robust and accurate segmentation of iris images using optimal partitioning," Proc. - Int. Conf. Pattern Recognit., vol. 4, pp. 578-
$581,2006$.

[21] T. H. Min and R. H. Park, "Comparison of eyelid and eyelash detection algorithms for performance improvement of iris recognition," Proc. - Int. Conf. Image Process. ICIP, pp. 257-260, 2008

[22] P. Perona and J. Malik, "Scale-space and edge detection using anisotropic diffusion," IEEE Transactions on Pattern Analysis and Machine Intelligence, vol. 12, no. 7. pp. 629-639, 1990.

[23] CASIA iris image database: http://www.sinobiometrics.com.

[24] Y. Yin, L. Liu, and X. Sun, "SDUMLA-HMT: A multimodal biometric database," Biometric Recognition. Springer, Berlin Heidelberg, pp. 260-268, 2011.

[25] L. Masek, "Recognition of human iris patterns for biometric identification," Bachelor thesis, University of Western Australia, Australia, 2003.

[26] L. W. Liam, M. Chekima, L. C. Fan and J. Dargham, "Iris recognition using self-organizing neural network," In Research and Development, SCOReD, Student Conference on, pp. 3-6, 2002

[27] M. Mahlouji and A. Noruzi, "Human Iris Segmentation for Iris Recognition in Unconstrained Environments," IJCSI International Journal of Computer Science, vol. 9, no. 1, pp. 149155, 2012.

[28] H. Raida, M. Boussellmi, and M. Abid, "Study the iris segmentation method for biometric pattern recognition," Proc. 3rd Int. Conf. Next Generation Networks and Services, pp. 108$111,2011$.

[29] G. Xu, Z. Zhang, and Y. Ma, "Automatic iris segmentation based on local areas," Proc.-Int. Conf. Pattern Recognit., vol. 4, no. c, pp. 505$508,2006$. 